\title{
Electroerosion formation and technology of cast iron coatings on aluminum alloys
}

\author{
Vladislav P. Smolentsev ${ }^{1}$, Oleg N. Fedonin ${ }^{2}$, and Eugene V. Smolentsev ${ }^{1, *}$ \\ ${ }^{1}$ Voronezh State Technical University, 14, Moskovsky Ave., Voronezh, 394026, Russia \\ ${ }^{2}$ Bryansk State Technical University, 7, 50-letiya Octyabrya Blvd, Bryansk, 241035, Russia
}

\begin{abstract}
At present in the course of designing basic production parts and industrial equipment designers pay more and more attention to aluminum alloys having a number of properties compared favorably with other materials. In particular, technological aluminum tool electrodes without coating in the presence of products of processing with alkali in the composition of operation environment are being destroyed at the expense of intensified material dissolution. It is shown in the paper that the method offered by the authors and covered by the patents on cast iron coating of products made of aluminum alloys, allows obtaining on a product surface the layers with high adhesion durability ensuring a high protection against destruction in the friction units including operation in hostile environment. Thereupon, aluminum, as compared with iron-based alloys used at manufacturing technological equipment for electrical methods of processing, has a high electrical and thermal conduction, its application will allow achieving considerable energy-saving in the course of parts production. A procedure for the design of a technological process of qualitative cast iron coatings upon aluminum tool electrodes and parts of basic production used in different branches of mechanical engineering is developed.
\end{abstract}

\section{Introduction}

In industry there has been developing a new direction of operation characteristics increase in products by means of a surface layer modification including the metal coatings corresponding to the requirements of new equipment designers. In such a way, in aerospace industry one tries to combine in one object of production different operational requirements which were considered earlier to be unrealizable [10-13]. One of these objects represents the parts made of light aluminum alloys having high wearresistance, resistance to hostile environment, for example, due to cast iron coatings. In recent years encouraging results on electroerosion coatings made of materials with a high melting point (for example, cast iron) are obtained. For manufacturing a qualitative layer it was necessary that an inter-electrode gap control should be used where an electrode vibrates along the axis with the frequency of 50$120 \mathrm{~Hz}$. At the same time, low-frequency oscillations of the tool must be coordinated with the process of coating as without reciprocal tool motions an aluminum alloy melts under coating applied and the part geometry is destroyed.

\section{Mechanism of coating}

Of great importance at the definition of quality parameters in the coating applied (for example, hardness, coating wear-resistance, adhesion properties and others) is a depth of the surface layer changed in which thermal and diffusion processes take place $[1,2]$, defining the heat and mass transfer (when applying cast iron coatings upon an aluminum basis the thermal changes and also the doping of aluminum with carbon, iron and other elements are possible). The diagram of the process of the surface layer formation and metal coating is presented in Fig. 1.

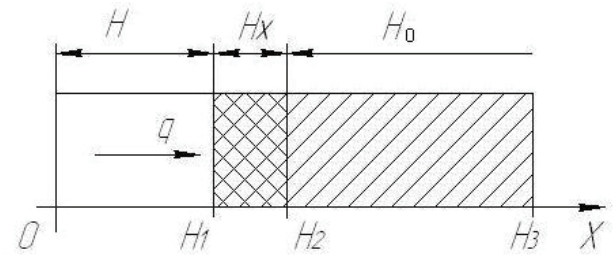

Fig. 1. The structure of the coating layer: $H$ - layer of metal applied; $H_{x}$ - molten layer; $H_{0}$ - blank metal; $q$ - heat flow coming into surface layers of the area of blank coating.

The Figure shows a dynamics of coating formation. A high-temperature metal drop (for example, cast iron) torn off from the anode and moving through a discharge channel falls onto heat-resisting oxide film on the surface of metal processed with a low melting point the initial temperature of which is $T_{0}$. Without flux presence after the contact of a drop with a coating area, a film heating and a blank sub-surface layer reflow occur. As a result, a melt layer of variable quantity $H_{x}(\tau)$, which disturbs blank accuracy and surface layer quality, is formed. It is supposed that the process should proceed at the vibration

* Corresponding author: smolentsev.rabota@gmail.com 
of a tool electrode with the frequency recommended, for example, in the work [3].

A new method [4] is offered for the cast iron coatings with the surface preparation in the course of the combined processing by means of the chemical removal of a hightemperature oxide film preventing the production of qualitative cast iron layers [5]. At the heart of this method lie the heat- and mass transfer and heat insulator removal from the surface of a blank during the coating application.

In the study of the process of the heat- and mass transfer during the alloying and coating

the dynamics of melting boundary motions into material of a blank and a possibility of the element diffusion of metal coated in the melt bath and a coating and also changes of diffusion coefficients and thermal physical characteristics in the discharge period were taken into account.

At that, the following assumptions were accepted: - the pressure in the discharge channel is accepted to be equal to the atmospheric one that corresponds to reality only at the initial stage of the discharge. Inasmuch as the duration of each discharge does not exceed some microseconds, then such an assumption is quite substantiated; - as a result of the breakdown of the inter-electrode gap, plasma is not formed, though it concerns only separate stages of the pulse. Here, at the expense of the mass transfer, plasma moves away from the coating applied and cannot affect substantially the process;

- the blank surface melting is carried out without taking into account the evaporation and metal atmospheric emission from the surface of heating;

- the problem solution was carried out in the Cartesian coordinate system the beginning of which coincides with the external boundary of the layer of the coating applied.

$$
\begin{gathered}
\frac{\partial C_{1}}{\partial \tau}=\frac{\partial}{\partial x}\left(D_{1} \frac{\partial C_{1}}{\partial x}\right), \quad 0 \leq x \leq H_{1}, \\
c_{1} \rho_{1} \frac{\partial T_{1}}{\partial \tau}=\frac{\partial}{\partial x}\left(\lambda_{1} \frac{\partial T_{1}}{\partial x}\right), \quad 0 \leq x \leq H_{1}, \\
c_{2} \rho_{2} \frac{\partial T_{2}}{\partial \tau}=\frac{\partial}{\partial x}\left(\lambda_{2} \frac{\partial T_{2}}{\partial x}\right)+\frac{Q_{m e l} \cdot W}{H_{2}}, \quad H_{1} \leq x \leq H_{3},(3) \\
\frac{\partial C_{2}}{\partial \tau}=\frac{\partial}{\partial x}\left(D_{2} \frac{\partial C_{2}}{\partial x}\right), \quad H_{1} \leq x \leq H_{2}
\end{gathered}
$$

where $c_{1}, c_{2}$ - heat capacity; $\rho_{1}, \rho_{2}-$ density; $T_{1}, T_{2}-$ temperature; $\lambda_{1}, \lambda_{2}$ - thermal-conductivity coefficients; $\tau$ - time; $x$-coordinate; $H_{1}, H_{2}, H_{3}$ - thickness of the layer coated, that of the molten one and a layer of the main metal accordingly; $C_{1}, C_{2}$ - mass concentration of anode elements in the coating and in molten metal; $D_{1}, D_{2}-$ diffusion coefficients; $Q_{m e l}$ - melting heat of an aluminum alloy; $W=\rho_{2} V$ - mass speed of melting; $V$ - linear melting rate. Indices 1 and 2 correspond to metal of the anode and that of the cathode (of a blank).

Diffusion coefficients $D_{1}$ and $D_{2}$ are defined through the exponential dependence on temperature [6]

$$
\begin{aligned}
& D_{1}(T)=A_{1} \exp \left(-\frac{E_{1}}{R T_{1}}\right), \\
& D_{2}(T)=A_{2} \exp \left(-\frac{E_{2}}{R T_{2}}\right),
\end{aligned}
$$

where $A_{1}$ и $A_{2}$ - pre-exponential multipliers (factors of the metal diffusion in a coating and a base); $E_{1}$ and $E_{2}-$ activation energy; $R$ - gas constant.

Initial conditions (at $\tau=0$ ):

$$
\begin{gathered}
T=T_{0}, \\
C=C_{01} \text { at } 0 \leq x \leq H_{1}, \\
C=C_{02} \text { at } H_{1} \leq x \leq H_{3}, \\
H_{x}=0,
\end{gathered}
$$

where $T_{0}$ - initial temperature; $C_{01}$ and $C_{02}$ initial concentration of anode elements in a coating and in melt (micro-bath); $H_{x}$ - molten layer thickness.

Boundary conditions:

$$
\begin{gathered}
\frac{\partial T_{1}}{\partial x}=0 \text { at } x=0 \\
\lambda_{2} \frac{\partial T_{2}}{\partial x}=q+q_{*} \quad \text { at } x=H_{1}, T_{2}=T_{m e l}, \\
\frac{\partial T_{2}}{\partial x}=0 \text { at } x=H_{3} \\
C=C_{1} \text { at } x=0 \\
D_{1} \frac{\partial C_{1}}{\partial x}=D_{2} \frac{\partial C_{2}}{\partial x} \text { at } x=H_{1} \\
\frac{\partial C_{2}}{\partial x}=0 \text { at } x=H_{2}
\end{gathered}
$$

The expression (12) is a boundary condition of the second kind which describes body heating by two sources of heat.

The first source - part of heat from electro-spark discharges coming into surface layers of the blank worked $-q$.

The second source $q_{*}$ - heat transferred by metal applied of the electrode.

At that $q$ and $q_{*}-$ are defined through the formulae

$$
\begin{gathered}
q=\frac{P_{a v}}{S} \cdot K, \\
q_{*}=L_{f e} \cdot m_{\kappa} .
\end{gathered}
$$

Here $S$ - electrode cross-sectional area (under condition that the diameter of the discharge channel is close to the diameter of an electrode operation part which corresponds to a tapered end of the tool taking into 
account its wear); $L_{f e}$ - latent heat of the electrode material melting; $m_{\kappa}$ - mass of the drop of material deposited on a blank during one pulse.

The average power of the generator $\left(P_{a v}\right)$ estimated through average current $\left(I_{a v}\right)$ and voltage $\left(U_{a v}\right)$

$$
P_{a v}=U_{a v} I_{a v}
$$

Factor $K$ in (17) characterizes the part of pulse energy consumed for heating a blank surface:

$$
K=1-\frac{2}{p+1}
$$

where $p$ - coefficient of thermal activity depending upon the thermal conductivity, heat capacity and density of electrode materials:

$$
p=\sqrt{\frac{\lambda_{1} c_{1} \rho_{1}}{\lambda_{2} c_{2} \rho_{2}}}
$$

The system of obtained non-linear differential equations of thermal conductivity and diffusion for coating metal and metal for a blank with non-linear boundary conditions on the surface of the environment division was being solved through the method of finite differences.

The numerical investigations were carried out in the range of the parameters of outer effects and characteristics of the transfer $[6,7]$ typical for the modes of electroerosion metal coatings.

To solve the system of equations it is necessary to know the thickness of the molten layer, $H_{x}$ the numerical value of which is calculated at any point of time through the formula:

$$
H_{x}(\tau)=h\left(i(\tau)+\frac{T_{i-1}(\tau)-T_{m e l}}{T_{i-1}(\tau)-T_{i}(\tau)}\right),
$$

where $h$ - pitch of a differential grid by $x ; i$ - number of a mesh point in which a temperature value achieved a metal melting temperature at a certain point of time; $T_{i-1}, T_{i}-$ temperature value at the same point of time in $(i-1)$ and $i$-th points of a grid; $T_{m e l}-$ melting temperature of a metal basis.

By (23) it is possible to calculate a linear melting rate:

$$
V=\frac{H_{x}(\tau+\Delta \tau)-H_{x}(\tau)}{\Delta \tau},
$$

where $H_{x}(\tau+\Delta \tau)$ and $H_{x}(\tau)$ - thickness of a molten layer at the point of time under consideration and at a previous point of time; $\Delta \tau-$ pitch of a differential grid in time.

In the course of the fulfillment of numerical computations the following parameter values were used [6]: $c_{1}=500 \mathrm{~J} /\left(\mathrm{kg}{ }^{0} \mathrm{~K}\right) ; c_{2}=250 \mathrm{~J} /\left(\mathrm{kg}{ }^{0} \mathrm{~K}\right) ; \rho_{1}=7100$ $\mathrm{kg} / \mathrm{m}^{3} ; \rho_{2}=2800 \mathrm{~kg} / \mathrm{m}^{3} ; \lambda_{1}=(46 \div 50) \mathrm{W} / \mathrm{m}^{\cdot 0} \mathrm{~K} ; \lambda_{2}=140$ $\mathrm{W} / \mathrm{m} \cdot{ }^{0} \mathrm{~K} ; T_{m e l}=930{ }^{0} \mathrm{~K} ; T_{0}=300{ }^{0} \mathrm{~K} ; S=(2 \div 4) \mathrm{mm}^{2} ; L_{f e}$ $=217,72 \mathrm{~kJ} / \mathrm{kg} ; Q_{m e l}=390 \mathrm{~J} / \mathrm{g} ; E_{1}=4806 \mathrm{~kJ} / \mathrm{mol} ; E_{2}=$ $125 \mathrm{~kJ} / \mathrm{mol} ; C_{01}=(92,8 \div 94,6) \%(\mathrm{Fe}) ; C_{02}=(90,5 \div 92,9)$ $\%$.

$P_{a v}$ and $m_{\kappa}-$ defined through a computational method.

\section{Technological process designing}

The analyzed mechanism of a surface layer formation allows carrying out the computations of basic parameters of machining modes, taking into account the peculiarities of the processes of coating under the flux layer and developing the recommendations for the material selection for manufacturing a part and a tool electrode shown in [5]. On the basis of this a procedure for the design of a technological process of cast iron coating upon aluminum alloys under a flux layer by means of an electroerosion method is developed.

The paper reports the consideration of two basic stages of the process designed [7]: the preparatory stage and the production one, including the part coating process consisting mainly of operations of the coating layer formation by the electroerosion method.

In the preparatory phase the following is carried out:

1) the selection substantiation of aluminum alloy parts, requiring cast iron coating or that of other materials with a high melting point, ensuring the required operation properties of the product;

2) the assessment of possibility to achieve the required technological indices calculated, according to the dependences shown at the description of the mechanism of coating formation; here some options are possible:

a) basic operation requirements are obtainable and a technological process should be designed for its realization;

b) at the current moment, some requirements cannot be obtained, but for the rest of indices a new process is efficient technically and economically. Here it should be expedient to introduce some precise definitions into a technical specification or technical requirements in a detail drawing and to design a technological process;

c) the specified requirements to a product are still unattainable and one has to pass to other kinds of materials and technological processes;

3) at the same stage, the improvement of the manufacturability of the part design is carried out in the course of which it is necessary to take into account: the character of production, dimensions of parts, physicalengineering properties of aluminum alloys, their peculiarities in behavior in the course of coating application, an error value of a real size, the specified technical requirements to coatings;

4) (if necessary) one improves the geometry of a part, taking into account further machining, calculates machining allowance and obtainable thickness of a qualitative layer obtained;

5) the choice and preparation of means for the technological equipment are carried out: as a rule, all devices used in the course of parts production may be chosen from the number of those used under conditions of a repetition work (at small dimensions of parts there is no need in the selection of handling machinery, which simplifies considerably equipment design and reduces its manufacturing cost);

6) the geometry and material are chosen for a tool electrode. For coating it is recommended to use a rod tool electrode made of cast iron of SCh20 kind. The application of cast iron surface layers for the purpose of 
corrosion protection is most efficient for electrodes for electrical-chemical treatment in alkaline medium and in environment containing chloride compounds in which aluminum alloys are destroyed quickly. The operation properties of such coatings, in addition to physical, mechanical and chemical characteristics of electrode material used, depend upon the reasonableness of a mode choice and technological process perfection [5]. At the same time, besides structure, properties and chemical composition of material of the coating obtained a significant criterion is smoothness of the coating, its thickness, a diffusion layer depth ensuring a durable adhesion of the coating with the basis and so on [5];

7) the working environment should be chosen and its composition is to be defined. The compatibility of the electrode material and working environment is a significant factor defining not only the efficiency of the process, but also a possibility of its fulfillment. Authors of many works emphasized the dependence of the character of the anode material application on cathode of the chemical interaction of electrode material with elements of inter-electrode environment [8], [9]. It is offered to use as a working medium the flux of FS-71 type having activating properties at coating upon aluminum alloys by the electroerosion method. In the course of processing, the flux enters the reaction with a surface oxide film $\mathrm{Al}_{2} \mathrm{O}_{3}$ destroys it, dissolves partially and removes it as a suspension cleaning, in such a way, the surface of a blank against a film, which not only prevents a stable process, but is often a main reason in the destruction of a cathode surface;

8 ) the modes of electroerosion coating are established [5;7], the quantity of layers essential for the formation of a coating with the quality required is defined. It is recommended to apply not less than three coating layers. At that it should be taken into account that the application of the second layer causes roughness increase, but increases heat-resistance and smoothness of the layer formed.

9) the formation of the technological process and in accordance with this, the manufacturing (the second stage of designing) of aluminum alloy parts with cast iron coating is carried out.

On the basis of the route mentioned above a procedure for the technological process designing taking into account the specificity of the coating under consideration which includes parts control on the whole and surfaces subject to processing in particular is formed. A possibility of processing is formed, a real value of the parameter after working is defined, a conformance to an error value is checked up.

At the second (manufacturing) stage the preparation of a blank surface for coating is carried out. When preparing a surface for coating there should be removed stains of dirt, paint, oils and fats. The operation is to be carried out by mechanical purification, rinse, degreasing and etching. An area joining the surface worked with the width of $(25$ $\div 30$ ) mm is subject to cleaning. At that the application of abrasive tools for this purpose is unacceptable as crumbled abrasive particles introducing inculcating into a surface can form various inclusions affecting adversely the quality of a coating performed.
Degreasing is carried out with the aid of organic solvents: gasoline, acetone. Parts degreased are washed out in lukewarm water (temperature not low than $298 \mathrm{~K}$ ) and rubbed clean with a stiff bristle brush. Etching should be carried out in $15-\%$ aqueous solution of nitric acid at the temperature of $(333 \div 338) \mathrm{K}$ during $(2 \div 5) \mathrm{min}$ or in $30 \%$ aqueous solution of nitric acid at the temperature of $(293 \div 298) \mathrm{K}$ during $(1 \div 2)$ min to neutralize remains of alkali, after that the parts should be washed out in lukewarm water.

Further, the improvement of modes is fulfilled on the equipment and manufacturing a sample with the aid of which the control of coating quality and the assessment of process efficiency is carried out.

On the basis of the results obtained in the course of the fulfillment of control operations the correction of modes and working a basic work-pieces batch are carried out.

The computations and experiments performed have shown that at electroerosion cast iron coating (material SCh-20) on aluminum alloy (Material D16) a rational combination of modes and conditions of treatment carrying out are the following:

- $A_{\text {pulse }}=(0,1 \div 0,5) \mathrm{J}$

- $U_{a v}=(50 \div 60) \mathrm{V}$

- $I_{\text {short circuit }}=(3 \div 5) \mathrm{A}$;

- $\tau_{\text {pulse }}=\left(10^{2} \div 10^{3}\right) \mu \mathrm{s}$

- feed speed of "electrode-tool" $V_{e}=(50 \div 200)$ $\mathrm{mm} / \mathrm{min}$;

- number of layers $n=3$;

Coating is carried out during the period of time essential for the formation of the coating with the thickness required, $T_{0}=(3 \div 5) \mathrm{min} / \mathrm{cm}^{2}$.

\section{Application of coatings for production of technological equipment and parts of basic production}

A general scheme of the operation sequence in the technological process is realized at manufacturing a tool electrode made of aluminum alloy; an assembly drawing and a drawing of the aluminum flange with coating are shown in Fig. 2: a) general view: 1 - aluminum alloy flange with cast iron coating for the tool electrode mounting, the fulfillment of current supply and electrolyte supply (place "a"); 2 - transition mandrel; 3 - electrically insulating dielectric coating; 4 - working element of the aluminum alloy tool electrode with cast iron coating; $b$ ) external view and basic dimensions of a flange.

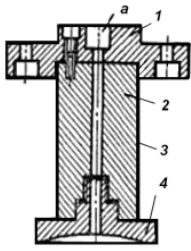

a)

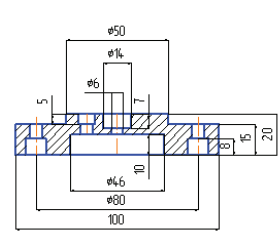

b)

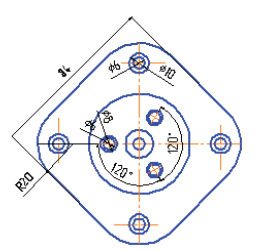

)
Fig. 2. The composite tool electrode for the combined erosionchemical treatment 
The increased working capacity of the coatings mentioned is ensured by the absence of a wedging effect under conditions of liquid friction and also by a specific profile characterized by a round micro-profile affecting in a positive way a load distribution.

The properties mentioned above and also a high durability of such coatings under the influence of electric current of high density and an electrolyte flow make their application possible as protective ones at manufacturing baths, cisterns, current leads and other elements of technological equipment operating in the modes of cavitation liquid flow at a long term of operation, for example, at combined erosion-chemical treatment.

The application of the developed method of coating for the corrosion resistance increase of surfaces in parts subjected to the impact of hostile working medium is also promising. The external view of some parts is presented in Fig. 3.

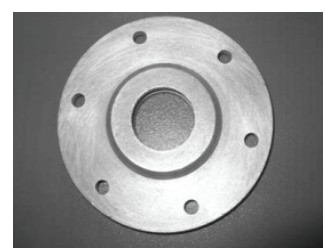

a)

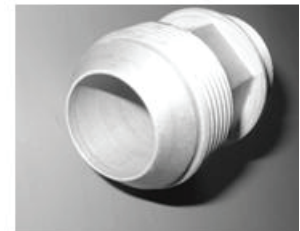

b)
Fig. 3. The external view of aluminum alloy parts which are to be coated with cast iron under a flux layer: $a$ - flange; $b$ connection

Before coating, the parts (Fig.3) made of material VD17 are subjected to machining, the accuracy control of geometrical parameters and the mutual location of surfaces, after that the layers of cast iron coating SCh20 with the general thickness, $0,4+0,1 \mathrm{~mm}$ are applied on the surface of the side of the mating areas. After that the surface coated is ground with allowance removal of 0.2$0.22 \mathrm{~mm}$ and the part becomes resistant to wear and durable to hostile medium impact.

\section{Conclusion}

The physical model and the mechanism of electroerosion coating are formed taking into account the necessity of low-temperature oscillations of a tool electrode, the specificity of the formation of high temperature surface layers on aluminum alloys under a layer of chemically active flux.

The system of indices essential for designing technological processes [7] for high temperature coatings on the surface of parts made of aluminum alloys by the electroerosion method is developed.

It is shown that the application of parts with a coating allows attaining the efficient protection against wear and hostile medium impact at the reduction of product mass, material electrical conductivity increase at the expense of its substitution and decreasing more than twice power consumption at its supply for processing.

\section{References}

1. A.V. Perova, Proceedings of OrelSTU, 4(272), 8 (2008)

2. V.P. Smolentsev, O.N. Kirillov, S.V. Dultsev, M.V. Shchipanov, Patent 2318637 (The Russian Federation), Bulletin 7 (2008).

3. G.P. Teslin, Soldering with Vibration Application (L., LDNTP, 1967)

4. V.P. Smolentsev, A.V. Grebenshchikov, A.V. Perova, B.I. Omigov, Patent 2405662 (The Russian Federation), Bulletin 34 (2010)

5. A.V. Perova, V.P. Smolentsev, A.S. Gribanov, Aerospace Techniques, 2008.

6. A.P. Eichis, B.Ya. Temkina, Technology of Surface Treatment of Aluminum and Its Alloys (M.: Machgiz, 1963)

7. E.V. Smolentsev, Design of Electrical and Combined Methods of Treatment (M., Mechanical Engineering, 2005)

8. O.N. Krakhmalyov, D.I.Petreshin, O.N. Fedonin, Modern problems of theory of machines, 4, 78 (2016)

9. O.N. Krakhmalyov, D.I.Petreshin, O.N. Fedonin, IOP: Conference Series. Materials Science and Engineering, 124 (2015)

10. A.V. Khandozhko, D.I. Petreshin, O.N. Fedonin, V.B. Protasiev, N.A. Bizyakina, D.Yu. Shukin, IOP: Conference Series. Materials Science and Engineering, 124 (2015)

11. D.I. Petreshin, A.V. Khandozhko, O.N. Fedonin, IOP: Conference Series. Materials Science and Engineering, 124 (2015)

12. S.V. Safonov, E.V. Smolentsev, O.N. Fedonin, IOP: Conference Series. Materials Science and Engineering, 124 (2015)

13. V.P. Smolentsev, S.V. Safonov, E.V. Smolentsev, O.N. Fedonin, IOP: Conference Series. Materials Science and Engineering, 124 (2015) 